\section{Distortion of a Free Surface during Tank Discharge}

\author{
D. F. GLUCK, ${ }^{*}$ J. P. GILLe, $†$ E. E. ZuKoski, \\ AND D. J. SIMKIN $\S$ \\ North American Aviation, Downey, Calif.
}

\section{Introduction}

D URING the process of withdrawing fluids from tanks, surface distortion and subsequent gas ingestion have been observed in situations where no liquid rotation was present. The distortion takes the form of a depression of the liquid surface over the outlet, which leads to ingestion of gas in the outlet line before all of the liquid has drained from the tank. This phenomenon becomes more important as the fluid flow rate is increased.

The flow of fluids from tanks, under conditions that lead to gas ingestion, has been studied with emphasis on a theoretical solution, e.g., Abramson et al. and Saad and Oliver. However, no results applicable to the problem studied here have been obtained, and to the authors' knowledge, no comprehensive experimental study of the ingestion phenomenon has been undertaken prior to this work.

This paper describes the results of such an investigation for flat-bottomed, right cylindric tanks with right cylindric outlets located on the tank centerline. The work discussed here deals primarily with the determination of the maximum. height of the liquid surface when the surface distortion reaches the outlet. This distance is called the "gas ingestion" height, $h$. This note extends the experimental work and correlation of Ref. 3. A more complete description of the theoretical work is presented in Ref. 3 .

\section{Theoretical Considerations}

Certain conclusions can be drawn from examination of the time dependent differential equations for potential flow from a tank with a free surface close to the outlet. First, it appears that the free surface distortion can be expressed as a function of tank geometry through the ratio of tank diameter to outlet diameter $D / d$ and of the flow velocity through a Froude number $V^{2} / a d$. Here, the undistorted interface velocity in the tank is $V$, and the acceleration and/or gravitational field that gives rise to body forces in the liquid is $a$. Second, examination of the equations suggests that the gas ingestion height will increase and will approach asymptotically a limiting value as the Froude number increases.

Should surface tension or viscous forces be comparable to the dynamic pressure forces, then the influence of Bond number $\rho a D^{2} / \sigma$ and the Reynolds number $V D / \nu$ also will be important parameters. Here, $\rho, \sigma$, and $\nu$ are the density, surface tension, and kinematic viscosity of the liquid, respectively.

\section{Experiments}

Over 100 tests using flat-bottomed plastic cylinders with a centrally located outlet have been made. The model was

\footnotetext{
Received June 30, 1966

* Senior Research Engineer, Space and Information Systems Division. Member AIAA.

$\dagger$ Technical Specialist, Space and Information Systems Division. Member AIAA.

$\ddagger$ Consultant and Professor of Jet Propulsion, California Institute of Technology. Space and Information Systems Division. Associate Fellow ÁIAA.

$\S$ Manager, Propulsion, Systems, Research and Engineering, Space and Information Systems Division. Associate Fellow AIAA.
}

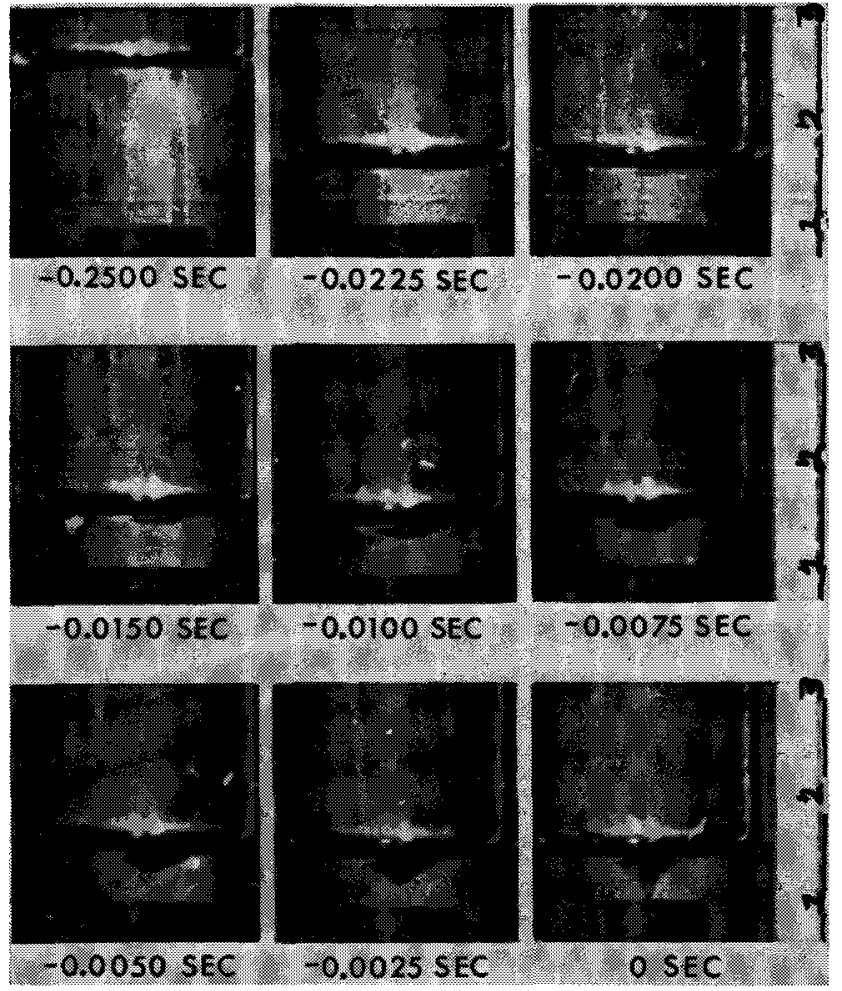

Fig. 1 Successive stages in development of gas ingestion for typical discharge.

pressurized with nitrogen gas and the pressure was always kept greater than 25 times the hydrostatic head of the test fluid. A large accumulator was provided between regulator and the model to minimize pressure surges during the short discharge period, and the tank inlet was baffled to prevent surface distortion caused by impingement of gas on the liquid surface. The velocity of the liquid-gas interface for each test was measured from photographic data obtained before the distortion of the interface became large. The height of the liquid near the wall, but outside the meniscus region, measured from the bottom of the tank, was obtained from the photographs at the instant that the gas reached the outlet. In a few tests, a 0.4-in. outlet was placed in an open tank 24$\times 48-\times 8$-in. deep tank; in these tests, the surface velocity essentially was zero.

The data were reproducible, and the gas ingestion heights were independent of the initial tank liquid level as long as this level was greater than $h$. Observations of the motion of bubbles and other impurities that were deliberately added showed no circulation of the liquid, and hence showed that vorticity is not responsible for the observed phenomena.

\section{Results and Analysis}

A photographic record of a typical water discharge from inception of interface distortion to gas ingestion is shown in Fig. 1. For this run, the fluid Froude number is 0.233 , the ratio $D / d$ is 10 , and the velocity of the undistorted interface is $4.2 \mathrm{in}$./sec. Time zero was arbitrarily taken as that when ingestion occurred.

The interface is flat to about $-0.02 \mathrm{sec}$, when interface distortion is first noted. In the next $0.02 \mathrm{sec}$, the interface at the centerline accelerates continuously, traveling the next 0.56 in. at an average rate 6.2 times greater than the velocity of the flat interface. The major distortion is confined to a small region about the centerline. 


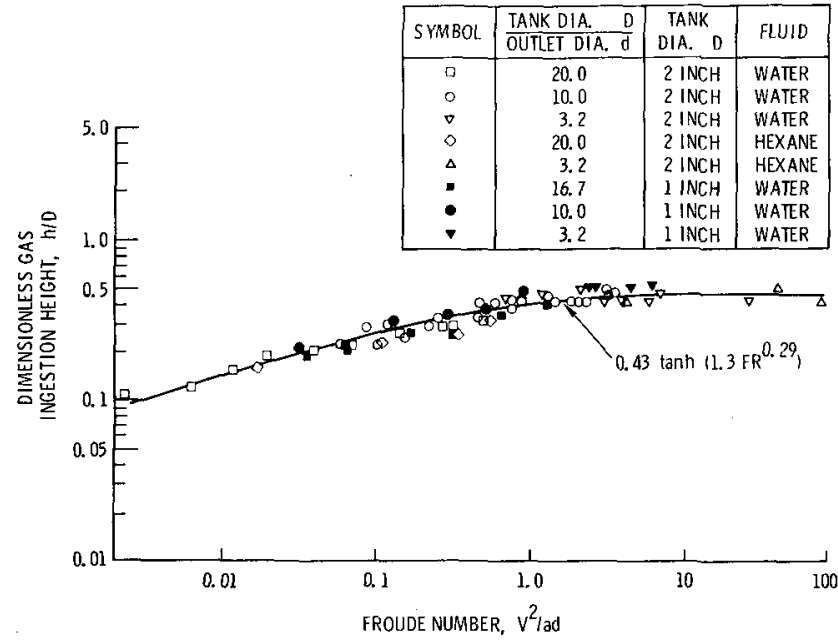

Fig. 2 Dependence of gas ingestion height on Froude number for various diameter ratios.

Analysis of the differential equations for the system studied here indicates that, in general, three dimensionless groups are required to describe the experimental results. One possible choice for these groups is $h / d=f\left(v^{2} / a d, D / d\right)$, where $v$ is the flow speed in the outlet line. However, examination of the experimental results shows that the data are well correlated by the one-parameter representation: $h / D=g\left(V^{2} / a d\right)=$ $g\left(F_{r}\right)$ and, to save space, the data are shown in terms of these variables in Fig. 2. The model sizes, diameter ratios, and fluids used are shown on the figure legend. The range of tank Froude numbers studied here was $10^{-3}<V^{2} / a d<$ $10^{2}$, which corresponds to the range $10^{-1}<v^{2} / a d<10^{5}$. In addition, the data cover a wide range of Reynolds and Bond numbers.

Generally, $h / D$ increases slowly with the Froude number until an asymptotic value of about 0.43 is reached at $V^{2} / a d$ $\approx 2.0$. For tanks with diameter ratios of 3.2 and 10 , this asymptote clearly is reached; for diameter ratios of 16.7 and 20 , the Froude numbers obtained are too low to show any asymptote. The data lie within $\pm 15 \%$ of an empirical correlation, $h / D=0.43 \tanh \left(1.3 F_{r}{ }^{0.29}\right)$.

For the few experiments carried out in the large open tank, $V^{2} / a d$ was always less than $4 \times 10^{-7}$ and $v^{2} / a d$ varied from 10 to 40 . The gas ingestion height increased with $v^{2} / a d$ and ranged from 0.75 to $1.0 \mathrm{~d}$.

For the data of Fig. $2, D / d$ ranged from 3.2 to 20 and the value of $h / D$ obtained at a given Froude number shows little systematic dependence on $D / d$. It is clear that the semiempirical correlation of the data leads to unreasonable predictions of $h / D$ in the limiting cases where $D / d$ approaches either one or infinity. A second correlation, $h / D=0.43$ $\tanh \left(F_{r}{ }^{0.25}\right)$, predicts values of $h / D$ which lie at most $10 \%$ below those given by the first correlation, and it gives a more satisfactory dependence of the gas ingestion height when $D / d \gg 1$. If one allows the diameter ratio to increase without bound while keeping the efflux rate constant, the tank Froude number approaches zero, and the second correlation reduces to: $h / d=0.43\left(v^{2} / a d\right)^{1 / 4}$. Thus, in the limiting case of $D \gg d$, the gas ingestion height becomes independent of the diameter ratio, and only depends on conditions in the outlet line. This equation is in excellent agreement with the data obtained in the large open-tank experiments, and hence the authors feel that the second correlation is most useful for low Froude numbers.

In order to separately ascertain the effect of Reynolds number on $h / D$, tests were run at several nearly constant Froude numbers in the range $0.5<\mathrm{Fr}<2$. Model diameter ratios were 10 and 3.2 , and fluid viscosities ranged from 1.0 to $700 \mathrm{cp}$. No systematic influence of Reynolds number was found for the range $10^{2}<V D / \nu<10^{5}$. Thus, the Reynolds number has, at most, only a minor influence on $h / D$ for these conditions.

The Bond number was changed from 90 to 900 for the data reported in Fig. 2, and no influence of the Bond number could be found. Some additional small-scale experiments were carried out in the Bond number range $90>\rho a D^{2} / \sigma>5$. For Bond numbers near 5, the steady-state liquid-gas interface was curved because of surface tension effects. Thus, in this case, it was necessary to measure $h$ from the extrapolated position of the undisturbed bottom of the meniscus. Given this definition of $h$, the measured values agree with the curve of Fig. 2, although the data are scattered $\pm 30 \%$. Hence, the effect of Bond number on $h$ appears to be unimportant for $\rho a D^{2} / \sigma>5$.

Studies on hemispherical-bottomed tanks also were carried out. Within the range where optical distortion was not excessive, little, if any, difference in gas ingestion heights because of the hemispherical bottom was noted.

\section{Conclusions}

The tests discussed here show that severe distortion of the surface of a liquid in an emptying tank can occur independent of and in the absence of any rotation of the liquid. The height of the liquid at the tank wall, when gas first reaches the tank outlet, has been determined for a wide range of parameters. When the ratio of tank diameter to outlet diameter lies between 20 and 3.2, this gas ingestion height is independent of the Reynolds number and Bond number for the ranges $10^{5}>V D / \nu>10^{2}$ and $\rho a D^{2} / \sigma<90$, and for these conditions depends only on a Froude number. For large tank Froude numbers, the gas ingestion height reaches the asymptotic value of $43 \%$ of the tank diameter, and for very small Froude numbers it depends on the $\frac{1}{4}$ root of an outlet line Froude number. The lower limits given herein on Reynolds and Bond numbers probably lie considerably above the values at which these parameters become important.

\section{References}

${ }^{1}$ Abramson, H. N., Wen-Hwa, C., Garza, L. R., and Ransleben, G. R., Jr., "Some studies of rotation and vortexing in rocket propellant tanks," NASA TN D-1212 (January 1962).

2 Saad, M. A. and Oliver, D. A., "Linearized time dependent free surface flow in rectangular and cylindrical tanks," Proceedings, Heat Transfer and Fluid Mechanics Institute (Stanford University Press, Stanford, Calif., 1964).

${ }^{3}$ Gluck, D. F., Gille, J. P., Simkin, D. J., and Zukoski, E. E., "Distortion of the liquid surface during tank discharge under low G conditions," Aerospace Chemical Engineering (American Institute of Chemical Engineers, New York, N. Y., 1966) Vol. 61. 